\title{
$H$-wise Independence*
}

\author{
Ishay Haviv \\ Michael Langberg ${ }^{\dagger}$ \\ Received January 8, 2018; Revised October 24, 2019, and in final form November 12, 2019; Published November
} 19,2019

\begin{abstract}
For a hypergraph $H$ on the vertex set $\{1, \ldots, n\}$, a distribution $D=\left(D_{1}, \ldots, D_{n}\right)$ over $\{0,1\}^{n}$ is $H$-wise independent if every restriction of $D$ to indices which form an edge in $H$ is uniform. This generalizes the notion of $k$-wise independence obtained by taking $H$ to be the complete $n$ vertex $k$-uniform hypergraph. This generalization was studied by Schulman (STOC 1992), who presented constructions of $H$-wise independent distributions that are linear, i.e., the samples are strings of inner products (over $\mathbb{F}_{2}$ ) of a fixed set of vectors with a uniformly chosen random vector.

Let $\ell(H)$ denote the minimum possible size of a sample space of a uniform $H$-wise independent distribution. The $\ell$ parameter is well understood for the special case of $k$-wise independence. In this work we study the notion of $H$-wise independence and the $\ell$ parameter for general graphs and hypergraphs. For graphs, we show how the $\ell$ parameter relates to standard graph parameters (e.g., clique number, chromatic number, Lovász theta function, minrank). We derive algorithmic and hardness results for this parameter as well as an explicit construction of graphs $G$ for which $\ell(G)$ is exponentially smaller than the size of the sample space of any linear $G$-wise independent distribution. For hypergraphs, we study the problem of testing whether a given distribution is $H$-wise independent, generalizing results of Alon et al. (STOC 2007).
\end{abstract}

\footnotetext{
*A preliminary version appeared in Proceedings of the 4th Innovations in Theoretical Computer Science (ITCS) conference, 2013, pages 541-552.

$\dagger$ Work supported in part by ISF grant 480/08 and by the Open University of Israel's research fund (grant no. 46114).
}

Key words and phrases: derandomization, $k$-wise independence, $H$-wise independence 


\section{Introduction}

In the past decades, the study of randomized algorithms has become a wide and rich field of research in theoretical computer science. Whereas randomization was found useful in many algorithmic applications, many efforts were made to understand the true role of randomness in computation. In many cases, it was shown that algorithms that use randomness can be deterministically simulated, i.e., derandomized.

In general, randomized algorithms can be viewed as two-step algorithms: in the first step, a sample point, consisting of $n$ random unbiased coins, is chosen according to the uniform distribution, and in the second step a deterministic algorithm is applied to the input and to the random coins from the first step. In many randomized algorithms the assumption that the random string of coins is chosen uniformly from all the $2^{n}$ possible random strings is not crucial for the analysis. Namely, it might suffice to take the random choice from a much smaller sample space. If it is possible to efficiently generate the elements of the smaller sample space then one can reduce the amount of randomness used by the algorithm. When the sample space is small enough, it is possible to deterministically apply the second step of the algorithm to every element in this space, and to decide according to the majority. Among other applications, this approach was found useful for derandomizing parallel algorithms, in which applying the deterministic algorithm with distinct samples can be done in parallel (see, e.g., [23, 3, 30, 31]). Therefore, research on the size of sample spaces with some randomness properties is motivated from theoretical and practical points of view.

The most fundamental type of randomness is pairwise independence, which means that every two coins are independent. It is well known that a sample space of size $O(n)$ suffices for picking $n$ pairwise independent unbiased coins. This was found useful in many applications, e.g., in constructions of efficient hashing schemes (for more applications see the survey of Luby and Wigderson [32]). The notion of pairwise independence is naturally generalized to $k$-wise independence for $k \geq 3$, in which the distribution on every induced $k$ bit locations of the random string is uniform. It was shown by Chor et al. [13] that every uniform $k$-wise independent distribution over $n$ bit strings has sample space of size $\Omega\left(n^{\left\lfloor\frac{k}{2}\right\rfloor}\right)$. Alon, Babai and Itai [3] extended this lower bound to the non-uniform case and gave a construction, which is based on $\mathrm{BCH}$ codes, of a uniform $k$-wise independent distribution matching this bound. This means that $k$-wise independent sample spaces of size polynomial in $n$ are only possible for constant $k$.

Several approaches were presented in the literature in order to overcome the lower bound of $[13,3]$. One idea was to consider a sample space of non-polynomial size and to use a conditional expectation technique in order to choose a good sample (instead of iterating over all the samples). This was used in $[33,8]$ to prove that certain problems lie in the complexity class NC. Another approach was taken by Naor and Naor [34] who introduced the notion of almost $k$-wise independence. Here, the restriction of the samples to $k$ coordinates should only be statistically close to a uniform distribution for some statistical distance parameter $\varepsilon$. It was shown in [34] (see also [4]) that the size of the sample space of such distributions can be significantly smaller than that of $k$-wise independent distributions and that almost $k$-wise independent distributions suffice for many applications (for more applications see, e.g., [1, 11]).

In this work we study another method, originally considered by Schulman [36], to overcome the lower bound on the size of sample spaces of $k$-wise independent distributions (see also [28]). To illustrate this method, let us look at the following very simple example. It is well known that for an $n$ vertex graph $G=(V, E)$ a random cut has expected size $\frac{|E|}{2}$, since every two adjacent vertices are in distinct 


\section{$H$-WISE INDEPENDENCE}

sides of the cut with probability $\frac{1}{2}$. Moreover, the same argument holds even if the cut partition is not chosen from a uniform distribution over all the $2^{n}$ partitions but from a pairwise independent distribution. However, the pairwise independence requirement might be in certain cases much stronger than what is really needed. Indeed, in order to have expected cut size $\frac{|E|}{2}$ all we need is a distribution over $\{0,1\}^{n}$ in which the coordinates corresponding to adjacent vertices are independent. We say that such a distribution is $G$-wise independent, and this is the notion of randomness considered in this paper. Notice that the randomness property required from the sample space depends on the input graph. It can be seen that a $G$-wise independent distribution might have much smaller sample space than a pairwise independent distribution. For example, it can be observed that if the graph $G$ is $k$-colorable for some constant $k$, a sample space of constant size suffices. The notion of $G$-wise independence is naturally extended to hypergraphs, where restrictions to indices which form an edge in the hypergraph should be uniform.

Definition 1.1 ( $H$-wise independence; $\ell(H))$. For an $n$ vertex hypergraph $H=([n], E)$, we say that a distribution $D=\left(D_{1}, \ldots, D_{n}\right)$ over $\{0,1\}^{n}$ is $H$-wise independent if for every edge $\left\{i_{1}, \ldots, i_{k}\right\} \in E$ all strings in $\{0,1\}^{k}$ are equally likely to occur in the restriction $\left(D_{i_{1}}, \ldots, D_{i_{k}}\right)$. A distribution $D$ is uniform $H$-wise independent if it is $H$-wise independent, and in addition it is uniform over a sample space $\Omega$ (i.e., choosing an element from $\Omega$ uniformly at random yields the distribution $D$ ). In this setting, $\Omega$ may be considered as a multiset of vectors in $\{0,1\}^{n}$. Let $\ell(H)$ denote the minimum possible size of a sample space $\Omega$ of a uniform $H$-wise independent distribution over $\{0,1\}^{n}$.

For example, for the 8-cycle $C_{8}$ it holds that $\ell\left(C_{8}\right)=4$ as one can define the distribution $D=$ $\left(D_{1}, D_{2}, \ldots, D_{8}\right)$ to be uniform over $\Omega=\{00000000,01010101,10101010,11111111\}$. Here for each edge $\{i, i+1\}$ in $C_{8}$ it holds that $D_{i}$ and $D_{i+1}$ are independent, where the indices are taken modulo 8. For the complete $n$ vertex $k$-uniform hypergraph $K_{n}^{(k)}$ we get the special case of $k$-wise independence, for which the $\ell$-value is known as stated below.

Theorem $1.2([13,3])$. For every $k, \ell\left(K_{n}^{(k)}\right)=\Theta\left(n^{\left\lfloor\frac{k}{2}\right\rfloor}\right)$.

The $\ell$ parameter given in Definition 1.1 measures the amount of randomness required by a randomized algorithm to handle a specific hypergraph instance $H$, thus its study may have implications on per-instance analysis of derandomization. In addition, Schulman presented in [36] several concrete applications motivating constructions of $H$-wise independent distributions. The applications of [36] include testing VLSI circuits, coding for burst errors on noisy channels, and derandomization of the algorithmic Lovász local lemma (for a progress on the latter see, e.g, [38]). We note that one can also formulate a variant of the $\ell$ parameter which generalizes almost $k$-wise independence, but in this work we take a first step in this line of research and concentrate on perfect independence.

The approach taken by Schulman [36] for constructing $H$-wise independent distributions for a $k$ uniform hypergraph $H$ is based on constructions of vectors over $\mathbb{F}_{2}$ with certain linear independence requirements. Namely, consider a multiset of $m$-dimensional vectors $\left\{v_{i}\right\}_{i=1}^{n}$ such that every set of vectors corresponding to an edge in $H$ is linearly independent. Let $D$ be the distribution defined by $D=\left(\left\langle w, v_{1}\right\rangle, \ldots,\left\langle w, v_{n}\right\rangle\right)$, where $w$ is uniformly chosen from $\mathbb{F}_{2}^{m}$ and $\langle\cdot, \cdot\rangle$ represents the inner product operator in $\mathbb{F}_{2}^{m}$. We refer to such distributions as linear. It is not hard to verify that $D$ is a uniform $H$-wise independent distribution whose sample space has size $2^{m}$, implying that $\ell(H) \leq 2^{m}$. Schulman [36] applied a simple probabilistic argument, which assigns uniformly chosen random vectors to vertices in the hypergraph $H$, to obtain the following bounds on $\ell$. 
Theorem 1.3 ([36]). Every $k$-uniform hypergraph $H=(V, E)$ satisfies $\ell(H) \leq 2^{k+1} \cdot|E|$. If every vertex of $H$ belongs to at most $\Delta$ edges then $\ell(H) \leq 2^{k+1} \cdot \Delta$. If every edge of $H$ intersects at most $\bar{\Delta}$ edges then $\ell(H) \leq 2^{k+1} \cdot e \bar{\Delta}$, where $e$ is the natural logarithm constant.

\subsection{Our Contribution}

In this paper we study several aspects of $H$-wise independence and the $\ell$ parameter given in Definition 1.1. Our contribution is two-fold. We first perform an extensive study of the $\ell$ parameter for graphs (i.e., 2-uniform hypergraphs), tying $\ell$ to several well-studied graph parameters. We then turn to address hypergraphs $H$, and focus our study on the problem of testing $H$-wise independence.

\subsubsection{The $\ell$ Parameter of Graphs}

We start with the special case of graphs and show how the $\ell$ parameter relates to several classical graph parameters. Denoting by $\omega(G)$ and $\chi(G)$ the maximum size of a clique in a graph $G$ and the chromatic number of $G$ respectively, we show that

$$
\omega(G) \leq \ell(G) \leq O(\chi(G))
$$

It is not difficult to observe that both the upper and lower bounds in (1.1) can be tight, as is achieved by the $n$ vertex complete graph $K_{n}$. Indeed, a $K_{n}$-wise independent distribution is pairwise independent, and the minimum possible size of a sample space of such uniform distribution is known to be $\Theta(n)[13,3]$. We turn to focus on the potential slackness in the upper bound of (1.1) and show that there are graphs whose $\ell$ parameter is significantly smaller than their chromatic number. Specifically, we present an explicit construction of a graph family for which the chromatic number is exponential in the $\ell$ parameter. This gap is proven to be optimal as stated below.

Theorem 1.4. For every graph $G, \ell(G) \geq \Omega(\log \chi(G))$. Moreover, for every sufficiently large $n$ there exists an explicit construction of an $n$ vertex graph for which this bound is tight.

The proof of Theorem 1.4 is based on a theorem due to Frankl and Rödl [18] which confirmed a 250\$ conjecture of Erdös [16] regarding the size of families of subsets of $\{1,2, \ldots, n\}$ with no two subsets intersecting at exactly $\frac{n}{4}$ elements (see Theorem 3.4).

Theorem 1.4 and its proof are used to obtain a couple of consequences. First, we show that there are graphs $G$ for which $\ell(G)$ is exponentially smaller than the size of the sample space of any linear $G$-wise independent distribution. This means that for certain graphs $G$ the approach taken by Schulman in [36] for constructing $G$-wise independent distributions inherently leads to a significant loss in the size of the sample space. Second, we obtain the following two negative computational results. (a) The problem of deciding, given a graph $G$, whether there is a uniform $G$-wise independent distribution with sample space of size $\ell$ is NP-complete for every constant $\ell$ divisible by 4 (see Theorem 3.13; notice that the size of the sample space is always divisible by 4 unless $G$ is edgeless). This follows from a characterization of the $\ell$ parameter in terms of graph homomorphisms and a result of Hell and Nešetřil on the hardness of a problem called $H$-coloring [21]. (b) Applying known hardness results on the chromatic number of a graph $[14,15]$, we derive that it is impossible to approximate the $\ell$ parameter of an $n$ vertex graph 


\section{$H$-WISE INDEPENDENCE}

in polynomial time to within a factor of $\Omega(\log \log n)$ assuming a certain variant of the Unique Games Conjecture of [26] (see Corollary 3.14).

A somewhat related graph parameter is the Shannon capacity of graphs, introduced by Shannon in [37]. The Shannon capacity of a graph $G$ is defined as $c(G)=\lim _{k \rightarrow \infty} \sqrt[k]{\alpha\left(G^{k}\right)}$, where $\alpha(G)$ stands for the independence number of $G$ and $G^{k}$ is what is known as the $k$-fold strong graph product of $G$ with itself. This graph parameter, studied in the context of information theory, measures the effective size of an alphabet in zero-error communication over a noisy channel represented by the graph. Unfortunately, the behavior of the Shannon capacity of graphs in general is far from being well understood. Even the Shannon capacity of very simple graphs, such as the cycle of length 7 , is not known. Therefore, upper and lower bounds on the Shannon capacity of graphs are of interest. It can be seen that the Shannon capacity of a graph satisfies $\alpha(G) \leq c(G) \leq \chi(\bar{G})$, or, equivalently, $\omega(G) \leq c(\bar{G}) \leq \chi(G)$. However, there are tighter upper bounds on the Shannon capacity. One well-known and tractable upper bound is due to Lovász [29], known as the Lovász $\vartheta$-function (see Definition 2.3). In this work we show that $\ell(G)$ is bounded from below by the $\vartheta$-function of the complement graph $\bar{G}$ implying that

$$
\omega(G) \leq c(\bar{G}) \leq \vartheta(\bar{G}) \leq \ell(G) \leq O(\chi(G)) .
$$

Using the above connections between the $\ell$ parameter, the Lovász $\vartheta$-function and the chromatic number, we employ the semidefinite programming algorithm of Karger, Motwani and Sudan [22] for graph coloring to obtain the positive algorithmic result stated below. ${ }^{1}$

Theorem 1.5. For every $\ell$ there exists a randomized polynomial-time algorithm that given an $n$ vertex graph $G$ satisfying $\ell(G)=\ell$ finds a linear $G$-wise independent distribution with sample space of size $\widetilde{O}\left(n^{1-\frac{3}{\ell+1}}\right)$.

Another upper bound on the Shannon capacity, called minrank, was introduced by Haemers in [20] (see Definition 2.4). The minrank and the Lovász $\vartheta$-function tend to behave quite differently and are known to be incomparable [19]. Nevertheless, similarly to the case of the $\vartheta$-function, we relate the $\ell$ parameter to the minrank and obtain that

$$
\omega(G) \leq c(\bar{G}) \leq \operatorname{minrk}_{\mathbb{R}}(\bar{G}) \leq \ell(G)+1 \leq O(\chi(G)),
$$

where $\operatorname{minrk}_{\mathbb{R}}$ stands for the minrank parameter over the reals.

\subsubsection{Testing $H$-wise Independence}

We now turn to study the problem of testing whether a given distribution over $\{0,1\}^{n}$ is $H$-wise independent for a general $n$ vertex $k$-uniform hypergraph $H .^{2}$ The goal is to develop an algorithm that given samples drawn from a distribution over $\{0,1\}^{n}$ with high probability accepts $H$-wise independent distributions and rejects distributions which are $\delta$-far in statistical distance from any $H$-wise independent distribution (uniform or not). We study the dependence of the number of queries required by such an algorithm on the hypergraph $H$. Previously this problem was addressed only for the special case of $H=K_{n}^{(k)}$, i.e., testing $k$-wise independence [5, 2, 35].

\footnotetext{
${ }^{1}$ The $\widetilde{O}$ and $\widetilde{\Omega}$ notations are used to hide poly-logarithmic factors.

${ }^{2}$ Throughout the discussion, for ease of presentation, $k$ is assumed to be a fixed constant and thus we do not explicitly state the (usually exponential) dependence of our expressions on $k$.
} 
Definition 1.6. Let $H$ be an $n$ vertex hypergraph, and fix some $\delta \in(0,1)$. An algorithm $A$ is a $\delta$-tester for $H$-wise independence with sample complexity $q$ if for every distribution $D$ over $\{0,1\}^{n}$, given an access to $q$ independently chosen samples from $D$,

- if $D$ is $H$-wise independent then $A$ accepts with probability at least $\frac{2}{3}$, and

- if $D$ is $\delta$-far from any $H$-wise independent distribution then $A$ accepts with probability at most $\frac{1}{3}$.

The approach taken in the literature for testing $k$-wise independence is based on calculating the biases of the drawn samples on all sets of at most $k$ bits. Since the biases measure the imbalance of exclusive-or on these sets, $k$-wise independent distributions are expected to have biases close to 0. However, for distributions that are far from $k$-wise independence, it was proved by Alon, Goldreich and Mansour [5] that at least one of these biases is expected to be bounded away from 0 . Their analysis implies that there is a tester for $k$-wise independence over $\{0,1\}^{n}$ with sample complexity $O\left(n^{2 k}\right)$. In a paper of Alon, Andoni, Kaufman, Matulef, Rubinfeld and Xie [2] the analysis was improved using Fourier transform techniques and led to an upper bound of $\widetilde{O}\left(n^{k}\right)$ on the sample complexity.

Considering $k$-wise independence as $H$-wise independence, where $H$ is the complete $n$ vertex $k$ uniform hypergraph $K_{n}^{(k)}$ with edge set $E_{n}^{(k)}$, the improved results of [2] can be stated in terms of the edge set size $\left|E_{n}^{(k)}\right|$. Namely, the tester of [2] for testing $K_{n}^{(k)}$-wise independence has sample complexity of $\widetilde{O}\left(\left|E_{n}^{(k)}\right|\right)$. In this work, we address general hypergraphs $H$, and present a tester for $H$-wise independence with sample complexity proportional to the number of edges in $H$. Our tester follows the line of that presented in [2] and calculates the biases of the samples on all subsets of edges in $H$. See Section 4.1 for details.

Theorem 1.7. For every $\delta>0, k$, and an $n$ vertex $k$-uniform hypergraph $H=([n], E)$ there exists a $\delta$-tester for $H$-wise independence with sample complexity $\widetilde{O}(|E|)$.

As for a lower bound on the sample complexity, it was implicitly shown in [5] that any tester of $k$-wise independence requires at least $\Omega\left(n^{k / 4}\right)$ queries. This followed from the observation that sample spaces of too small support must be far from $k$-wise independence. In [2, 39], a lower bound of $\Omega\left(\frac{1}{\delta} \cdot n^{\frac{k-1}{2}}\right)$ was shown for $\delta=O(1 / n)$ by studying the typical distance of a random uniform distribution from $k$-wise independence. Using our terminology and stating these results in terms of the edge set $E_{n}^{(k)}$ of the complete hypergraph $K_{n}^{(k)}$, one gets lower bounds of $\Omega\left(\left|E_{n}^{(k)}\right|^{1 / 4}\right)$ [5] and $\Omega\left(\frac{1}{\delta} \cdot \sqrt{\left.\left|E_{n}^{(k)}\right| / n\right)}\right.$ [2] accordingly.

In this work, we employ the techniques of $[5,2]$ aiming in obtaining a lower bound of $\Omega(\sqrt{|E|})$ for testing $H$-wise independence of general hypergraphs $H$ with edge set $E$. We present two incomparable results. Fitting the analysis of [2] to our setting, for small values of $\delta=O(1 / n)$, we present a lower bound of $\Omega\left(\frac{1}{\delta} \cdot \sqrt{|E| / n}\right) \geq \Omega(\sqrt{|E|})$. For general $\delta$, we enhance the techniques of [5] and show that any tester of $H$-wise independence must query at least $\Omega\left(\sqrt{\frac{n^{k}}{n^{k / 2}+|\bar{E}|}}\right)$ samples, where $|\bar{E}|$ is the number of non-edges of $H$. It is not hard to verify that the latter lower bound is at its best $\Omega\left(|E|^{1 / 4}\right)$, as achieved by very dense hypergraphs $H$, and is much weaker for sparser hypergraphs. See Section 4.2 for details. 
Theorem 1.8. For every $n$ vertex $k$-uniform hypergraph $H=([n], E)$, $\delta$-testing $H$-wise independence requires sample complexity at least $\Omega\left(\frac{1}{\delta} \cdot \sqrt{\frac{|E|}{n}}\right)$ if $\delta=O\left(\frac{1}{n}\right)$ and $\Omega\left(\sqrt{\frac{n^{k}}{|\bar{E}|+n^{k / 2}}}\right)$ if $\delta \leq \frac{1}{2}$ and $k$ is even. The same holds for an odd $k$ replacing $k$ by $k-1$ in the bound.

\subsection{Outline}

The paper is organized as follows. In Section 2 we gather all the definitions on distributions, graphs and Fourier transform needed in the paper. In Section 3 we present our results on the $\ell$ parameter of graphs and derive some algorithmic and hardness results. Finally, in Section 4 we prove upper and lower bounds on the sample complexity of testing $H$-wise independence for a general hypergraph $H$.

\section{Preliminaries}

\subsection{General}

We denote by $[n]$ the set $\{1,2, \ldots, n\}$. The Hamming weight of a vector $x \in \mathbb{F}_{2}^{n}$ is the number of its nonzero entries, and the Hamming distance between $x, y \in \mathbb{F}_{2}^{n}$, denoted $\operatorname{dist}_{H}(x, y)$, is the number of positions at which $x$ and $y$ differ. A probability distribution $D$ over $\{0,1\}^{n}$ is a function $D:\{0,1\}^{n} \rightarrow$ $[0,1]$ satisfying $\sum_{x \in\{0,1\}^{n}} D(x)=1$. The support of $D$ is defined as $\operatorname{support}(D)=\left\{x \in\{0,1\}^{n} \mid D(x) \neq\right.$ $0\}$. Let $U_{\{0,1\}^{n}}$ denote the uniform distribution defined by $U_{\{0,1\}^{n}}(x)=\frac{1}{2^{n}}$ for every $x \in\{0,1\}^{n}$. The statistical distance between two distributions $D^{(1)}$ and $D^{(2)}$ over $\{0,1\}^{n}$ is defined by $\Delta\left(D^{(1)}, D^{(2)}\right)=$ $\frac{1}{2} \cdot \sum_{x \in\{0,1\}^{n}}\left|D^{(1)}(x)-D^{(2)}(x)\right|=\max _{S \subset\{0,1\}^{n}} D^{(1)}(S)-D^{(2)}(S)$. The min-entropy of a distribution $D$ is defined as $-\log _{2} \max _{x \in\{0,1\}^{n}} D(x)$. For a distribution $D=\left(D_{1}, \ldots, D_{n}\right)$ over $\{0,1\}^{n}$, the bias of $D$ on a (non-empty) set $S \subseteq[n]$ is defined as

$$
\operatorname{bias}_{S}(D)=\operatorname{Pr}\left[\oplus_{i \in S} D_{i}=0\right]-\operatorname{Pr}\left[\oplus_{i \in S} D_{i}=1\right]=2 \cdot \operatorname{Pr}\left[\oplus_{i \in S} D_{i}=0\right]-1 .
$$

For an $n$ vertex hypergraph $H$ the distance of a distribution $D$ from $H$-wise independence, denoted $\Delta\left(D, \mathfrak{D}_{H-\text { wise }}\right)$, is defined as the minimum of $\Delta\left(D, D^{\prime}\right)$ over all $H$-wise independent distributions $D^{\prime}$ (see Definition 1.1). If $\Delta\left(D, \mathfrak{D}_{H \text {-wise }}\right) \geq \delta$ we say that $D$ is $\delta$-far from $H$-wise independence.

For a set system $E$ we denote by $\tilde{E}$ the set of all non-empty subsets of sets in $E$, i.e., $\tilde{E}=\{S|| S \mid \geq$ 1 and $\exists T \in E, S \subseteq T\}$. It is easy to see that for a hypergraph $H$ with edge set $E, H$-wise independence can be represented as a requirement on the biases on the sets in $\tilde{E}$. This is stated below.

Lemma 2.1. For every $n$ vertex hypergraph $H=([n], E)$ and a distribution $D$ over $\{0,1\}^{n}, D$ is $H$-wise independent if and only if bias $_{S}(D)=0$ for every $S \in \tilde{E}$.

\subsection{Graphs}

Let us recall several standard notions in graph theory. For a graph $G=(V, E)$, the independence number, denoted $\alpha(G)$, is the maximum size of a subset of $V$ containing no adjacent vertices. The clique number, denoted $\omega(G)$, is the maximum size of a subset of $V$ in which every two vertices are adjacent. A 
$k$-coloring of $G$ is a function $c: V \rightarrow[k]$ for which $\{x, y\} \in E$ implies $c(x) \neq c(y)$. The chromatic number of $G$, denoted $\chi(G)$, is the minimum $k$ for which $G$ is $k$-colorable.

A homomorphism from a graph $G_{1}=\left(V_{1}, E_{1}\right)$ to a graph $G_{2}=\left(V_{2}, E_{2}\right)$ is a function $f: V_{1} \rightarrow V_{2}$ which preserves edges, that is, $\{x, y\} \in E_{1}$ implies $\{f(x), f(y)\} \in E_{2}$. It is easy to see that if there is a homomorphism from $G_{1}$ to $G_{2}$ then $\chi\left(G_{1}\right) \leq \chi\left(G_{2}\right)$. A similar property is satisfied by the $\ell$ parameter (see Definition 1.1), as stated below.

Lemma 2.2. For every two graphs $G_{1}=\left(\left[n_{1}\right], E_{1}\right)$ and $G_{2}=\left(\left[n_{2}\right], E_{2}\right)$, if there is a homomorphism from $G_{1}$ to $G_{2}$ then $\ell\left(G_{1}\right) \leq \ell\left(G_{2}\right)$.

Proof. Let $f:\left[n_{1}\right] \rightarrow\left[n_{2}\right]$ be a homomorphism from $G_{1}$ to $G_{2}$ and let $D=\left(D_{1}, \ldots, D_{n_{2}}\right)$ be a $G_{2}$-wise independent distribution uniform on a multiset of size $\ell$. Consider the distribution of $D^{\prime}=\left(D_{f(1)}, \ldots, D_{f\left(n_{1}\right)}\right)$. Since $f$ preserves edges, $D^{\prime}$ is $G_{1}$-wise independent, hence $\ell\left(G_{1}\right) \leq \ell$.

We define below the Lovász $\vartheta$-function of a graph [29]. Note that the $\vartheta$-value of a graph is known to be equal to a graph parameter known as the strict vector chromatic number of the complement $\bar{G}$ of $G$ [22] (for many other equivalent definitions see [27]). It is well known that the $\vartheta$-value of a graph bounds its independence number from above, i.e., $\alpha(G) \leq \vartheta(G)$, and can be calculated in polynomial time using semidefinite programming.

Definition 2.3. An orthonormal representation of a graph $G=(V, E)$ is an assignment of a unit vector $a_{v}$ in a Euclidean space to each vertex $v \in V$ such that $\left\langle a_{u}, a_{v}\right\rangle=0$ for every distinct $u$ and $v$ satisfying $\{u, v\} \notin E$. The $\vartheta$-value of a graph $G$, denoted by $\vartheta(G)$, is equal to the minimum over all unit vectors $d$ and all orthonormal representations $\left(a_{v}\right)_{v \in V}$ of $G$ of $\max _{v \in V} \frac{1}{\left\langle d, a_{v}\right\rangle^{2}}$.

We now define the minrank parameter of a graph (over $\mathbb{R}$ ) which was introduced in [20]. We note that studying the minrank parameter over finite fields is motivated by a problem called index coding, as was shown by Bar-Yossef et al. [6].

Definition 2.4. Let $A=\left(a_{i j}\right)$ be an $n$ by $n$ real matrix. We say that $A$ represents an $n$ vertex graph $G=([n], E)$ if $a_{i i} \neq 0$ for all $i$, and $a_{i j}=0$ for every distinct $i$ and $j$ satisfying $\{i, j\} \notin E$. The minrank of a graph $G$ over $\mathbb{R}$ is defined as $\operatorname{minrk}_{\mathbb{R}}(G)=\min \left\{\operatorname{rank}_{\mathbb{R}}(A) \mid A\right.$ represents $\left.G\right\}$.

\subsection{Fourier Transform}

The set of functions from $\{0,1\}^{n}$ to $\mathbb{R}$ is a $2^{n}$-dimensional vector space in which the inner product of two elements $f$ and $g$ is defined by $\langle f, g\rangle=\frac{1}{2^{n}} \cdot \sum_{x \in\{0,1\}^{n}} f(x) \cdot g(x)$. For every set $S \subseteq[n]$ let $\chi_{S}$ : $\{0,1\}^{n} \rightarrow\{-1,1\}$ be the function defined by $\chi_{S}(x)=(-1)^{\sum_{i \in S} x_{i}}$. The set $\left\{\chi_{S} \mid S \subseteq[n]\right\}$ is known to be an orthonormal basis of the vector space, hence every function $f:\{0,1\}^{n} \rightarrow \mathbb{R}$ can be represented as $f=\sum_{S \subseteq[n]} \hat{f}(S) \cdot \chi_{S}$. The coefficients in this representation satisfy $\hat{f}(S)=\left\langle f, \chi_{S}\right\rangle$ and are called the Fourier coefficients of $f$. Observe that if $D$ is a distribution over $\{0,1\}^{n}$ then $\operatorname{bias}_{S}(D)=2^{n} \cdot \widehat{D}(S)$.

For every real $p \geq 1$ the $p$-norm of $f$ is defined by $\|f\|_{p}=\left(\frac{1}{2^{n}} \cdot \sum_{x \in\{0,1\}^{n}}|f(x)|^{p}\right)^{1 / p}$. For $p=\infty$, $\|f\|_{\infty}=\max _{x \in\{0,1\}^{n}}|f(x)|$. Parseval's equality is the standard name of the relation $\|f\|_{2}^{2}=\sum_{S \subseteq[n]} \hat{f}(S)^{2}$ which easily follows from the orthogonality of the basis. Observe that for two distributions $D^{(1)}$ and 
$D^{(2)}$ over $\{0,1\}^{n}, \Delta\left(D^{(1)}, D^{(2)}\right)=2^{n-1} \cdot\left\|D^{(1)}-D^{(2)}\right\|_{1}$. The following well-known hyper-contractive inequality will be used.

Theorem 2.5 (Beckner-Bonami Inequality [7, 10]). Let $f:\{0,1\}^{n} \rightarrow \mathbb{R}$ be a function that is a linear combination of $\left\{\chi_{S}|| S \mid \leq k\right\}$. Then, for every even $p>2,\|f\|_{p} \leq(p-1)^{k / 2} \cdot\|f\|_{2}$.

The convolution $f * g$ of two functions $f$ and $g$ is the function defined by

$$
(f * g)(x)=\frac{1}{2^{n}} \cdot \sum_{y \in\{0,1\}^{n}} f(y) \cdot g(x \oplus y)
$$

where $\oplus$ denotes the bit-wise addition, and is known to satisfy $\widehat{f * g}=\hat{f} \cdot \hat{g}$. A special case of Young's inequality says that $\|f * g\|_{\infty} \leq\|f\|_{\infty} \cdot\|g\|_{1}$.

\section{The $\ell$ Parameter of Graphs}

In this section we study the $\ell$ parameter (see Definition 1.1) for the special case of graphs and show how it relates to several graph parameters. We start with a definition of a graph family $G_{\ell}$ whose properties will be used in our proofs. The graphs $G_{\ell}$ are extremal with respect to our $\ell$ parameter, in the sense that for every graph $G$ with $\ell(G)=\ell$ there exists a homomorphism from $G$ to $G_{\ell}$ (see Claim 3.2).

Definition 3.1. For an $\ell$ divisible by 4 , the graph $G_{\ell}$ is defined as follows. Its vertex set consists of all the vectors in $\{0,1\}^{\ell}$ with Hamming weight $\frac{\ell}{2}$. Two such vectors $u, v$ are adjacent if they represent independent distributions, i.e., for every $(\alpha, \beta) \in\{0,1\}^{2}$ the number of indices $i$ for which $\left(u_{i}, v_{i}\right)=(\alpha, \beta)$ is $\frac{\ell}{4}$.

Observe that for every graph $G$ and every $\ell$ divisible by 4 , the following two statements are equivalent: (a) There exists a uniform $G$-wise independent distribution with sample space of size $\ell$. (b) There exists a homomorphism from $G$ to $G_{\ell}$. This equivalence follows from the natural correspondence between a uniform $G$-wise independent distribution with sample space of size $\ell$ and the homomorphism from $G$ to $G_{\ell}$ that maps every vertex to the $\ell$-dimensional vector in $G_{\ell}$ representing its distribution, where the $i$ th coordinate corresponds to the $i$ th sample (an isolated vertex is mapped arbitrarily). This gives the following alternative characterization of the $\ell$ parameter of graphs. To justify the restriction on $\ell$ stated below, we note that $\ell(G)$ is always divisible by 4 unless $G$ is edgeless.

Claim 3.2. For every non-edgeless graph $G, \ell(G)$ is the smallest $\ell$ divisible by 4 for which there exists a homomorphism from $G$ to $G_{\ell}$.

Using Lemma 2.2, we analyze the $\ell$-parameter of $G_{\ell}$ :

Lemma 3.3. For every $\ell$ divisible by $4, \ell\left(G_{\ell}\right) \leq \ell$. Moreover, if there exists a graph $G$ for which $\ell(G)=\ell$ then $\ell\left(G_{\ell}\right)=\ell$.

Proof. Let $n=\left(\begin{array}{c}\ell \\ \ell / 2\end{array}\right)$. Consider the distribution $D=\left(D_{1}, \ldots, D_{n}\right)$ over $\{0,1\}^{n}$ in which $D_{i}$ is exactly the distribution of support size $\ell$ implied by the $\ell$-vector corresponding to the $i$ th vertex of $G_{\ell}$. Now, for each edge $\{i, j\}$ of $G_{\ell}$ it holds that $D_{i}$ and $D_{j}$ are independent. Thus, $\ell\left(G_{\ell}\right) \leq \ell$. Moreover, let $G$ be any graph for which $\ell(G)=\ell$ then by Lemma 2.2 and Claim 3.2 it holds that $\ell=\ell(G) \leq \ell\left(G_{\ell}\right)$. 
We turn to estimate the chromatic number of $G_{\ell}$. We need the following theorem due to Frankl and Rödl [18] that confirmed a conjecture of Erdös [16].

Theorem 3.4 ([18]). There exists an absolute constant $c<1$ such that for every $n$ divisible by 4 and every family $\mathfrak{F}$ of subsets of $[n]$ without $F, F^{\prime} \in \mathfrak{F}$ such that $\left|F \cap F^{\prime}\right|=\frac{n}{4}$, it holds that $|\mathfrak{F}| \leq 2^{\text {cn }}$.

Lemma 3.5. For every $\ell$ divisible by $4, \chi\left(G_{\ell}\right)=2^{\Theta(\ell)}$.

Proof. On one hand, it is obvious that $\chi\left(G_{\ell}\right) \leq\left|V\left(G_{\ell}\right)\right|=\left(\begin{array}{l}\ell \\ \ell / 2\end{array}\right)<2^{\ell}$. On the other hand, observe that the vertices in every independent set of $G_{\ell}$ are the characteristic vectors of subsets of $[\ell]$ such that no two of them have intersection size $\frac{\ell}{4}$. To see this, observe that the characteristic vectors of every two subsets of $[\ell]$ of size $\frac{\ell}{2}$ that intersect at exactly $\frac{\ell}{4}$ elements must represent independent distributions. Hence, Theorem 3.4 implies that $\alpha\left(G_{\ell}\right) \leq 2^{c \ell}$ for some $c<1$, which implies that $\chi\left(G_{\ell}\right) \geq \frac{\left|V\left(G_{\ell}\right)\right|}{\alpha\left(G_{\ell}\right)} \geq \frac{(\ell / 2)}{2^{c \ell}}=$ $\frac{2^{(1-o(1)) \ell}}{2^{c \ell}}=2^{\Omega(\ell)}$.

Now, our lower and upper bounds on the $\ell$ parameter of graphs in terms of their chromatic number follow easily.

Theorem 3.6. For every graph $G, \Omega(\log \chi(G)) \leq \ell(G) \leq O(\chi(G))$. Moreover, there are explicit constructions of graphs attaining the upper and lower bounds for every sufficiently large number of vertices.

Proof. Observe that for every graph $G$, the function that maps every vertex to its color in an optimal coloring is a homomorphism from $G$ to $K_{\chi(G)}$. This is because every two adjacent vertices in $G$ are mapped to two distinct colors. By Lemma 2.2 and Theorem 1.2, $\ell(G) \leq \ell\left(K_{\chi(G)}\right)=O(\chi(G)$ ), and, as mentioned before, this bound is tight for the $n$ vertex complete graph.

For the lower bound, apply Claim 3.2 to obtain that every graph $G$ with $\ell(G)=\ell$ satisfies $\chi(G) \leq$ $\chi\left(G_{\ell}\right)<2^{\ell}$. By Lemma 3.3 and Lemma 3.5, the lower bound is attained at the graph family $G_{\ell}$ which satisfies $\ell\left(G_{\ell}\right) \leq \ell$ and $\chi\left(G_{\ell}\right)=2^{\Theta(\ell)}$.

As a consequence of Theorem 3.6, we show below that there are graphs $G$ for which $\ell(G)$, the minimum size of the sample space of a uniform $G$-wise independent distribution, is exponentially smaller than that of any linear $G$-wise independent distribution.

Corollary 3.7. For every sufficiently large $n$, there is an $n$ vertex graph $G$ for which $\ell(G) \leq O(\log n)$ whereas any linear $G$-wise independent distribution has sample space of size at least $n^{\Omega(1)}$.

Proof. Recall that for a graph $G=([n], E)$, a $G$-wise independent distribution is linear if it can be represented as $\left(\left\langle w, v_{1}\right\rangle, \ldots,\left\langle w, v_{n}\right\rangle\right)$ where the $v_{i}$ 's are fixed vectors in $\mathbb{F}_{2}^{m}$ for some $m$ and $w$ is uniformly chosen from $\mathbb{F}_{2}^{m}$. In this case the size of the sample space is $2^{m}$. Observe that the assignment of every vertex $i$ to the corresponding vector $v_{i}$ is a $2^{m}$-coloring of $G$. Indeed, if two vertices $i$ and $j$ are adjacent then the vectors $v_{i}$ and $v_{j}$ must be distinct. This implies that the chromatic number of $G$ is bounded from above by the size of the sample space of any linear $G$-wise independent distribution. Since the construction in Theorem 3.6 gives us an $n$ vertex graph $G$ with $\ell(G) \leq O(\log n)$ and $\chi(G) \geq n^{\Omega(1)}$ the result follows. 


\section{$H$-WISE INDEPENDENCE}

The following lemma relates the Lovász $\vartheta$-function of a graph (see Definition 2.3) to the $\ell$ parameter. Since every graph $G$ satisfies $\vartheta(G) \geq \alpha(G)$ [29], the lemma implies that for every graph $G, \ell(G) \geq \omega(G)$.

Lemma 3.8. For every graph $G, \ell(G) \geq \vartheta(\bar{G})$.

Proof. Consider a uniform $G$-wise independent distribution over $\{0,1\}^{n}$ with sample space of size $\ell$, and let $B$ denote an $\ell$ by $n$ Boolean matrix whose columns represent the vertex distributions. Namely, the $G$-wise independent distribution is obtained by choosing a row uniformly at random from $B$. For every vertex $v$ of $G$ let $b_{v}$ denote the column of $B$ indexed by the vertex $v$. Now, for every vertex $v$ define the unit $\ell$-dimensional vector

$$
a_{v}=\frac{1}{\sqrt{\ell}} \cdot\left((-1)^{\left(b_{v}\right)_{1}},(-1)^{\left(b_{v}\right)_{2}}, \ldots,(-1)^{\left(b_{v}\right)_{\ell}}\right) .
$$

Observe that $\left(a_{v}\right)_{v \in V}$ is an orthonormal representation of the complement $\bar{G}$ of $G$, since for every two vertices $u, v$ which are adjacent in $G$,

$$
\left\langle a_{u}, a_{v}\right\rangle=\frac{1}{\ell} \cdot\left(\ell-2 \cdot \operatorname{dist}_{H}\left(b_{u}, b_{v}\right)\right)=0 .
$$

Choosing $d=(1,0, \ldots, 0)$ we obtain that $\vartheta(\bar{G}) \leq \max _{v \in V} \frac{1}{\left\langle d, a_{v}\right\rangle^{2}}=\ell$.

Remark 3.9. It was shown in [22] (see also [17]) that there are $n$ vertex graphs $G$ satisfying $\vartheta(\bar{G})=3$ and $\chi(G) \geq n^{\Omega(1)}$. By Theorem 3.6, this implies that there are $n$ vertex graphs $G$ for which $\vartheta(\bar{G})=3$ and $\ell(G)=\Omega(\log n)$. In addition, a result of [17] implies that there are $n$ vertex graphs $G$ for which $\omega(G)<2^{\sqrt{\log n}}$ and $\ell(G)>n / 2^{O(\sqrt{\log n})}$.

We apply Lemma 3.8 to obtain the following algorithmic corollary.

Corollary 3.10. For every $\ell \geq 4$ there exists a randomized polynomial-time algorithm that given an $n$ vertex graph $G$ satisfying $\ell(G)=\ell$ finds a linear $G$-wise independent distribution with sample space of size $\widetilde{O}\left(n^{1-\frac{3}{\ell+1}}\right)$.

Proof. It was proved by Karger, Motwani and Sudan [22] that there exists a randomized polynomial-time algorithm that given an $n$ vertex graph $G$ such that $\vartheta(\bar{G}) \leq \vartheta$ for some $\vartheta \geq 2$ finds a coloring of $G$ that uses $\widetilde{O}\left(n^{1-\frac{3}{\vartheta+1}}\right)$ colors. Consider the algorithm that given an $n$ vertex graph $G$ satisfying $\ell(G)=\ell$ applies the algorithm of [22] to obtain a coloring of $G$. By Lemma 3.8, $(\bar{G}) \leq \ell$, hence this coloring uses at most $N=\widetilde{O}\left(n^{1-\frac{3}{\ell+1}}\right)$ colors. Denote the coloring by $c:[n] \rightarrow[N]$ and assume without loss of generality that $N=2^{k}-1$ for some integer $k$. Assign each color $i$ to a (distinct) nonzero vector $v_{i}$ in $\mathbb{F}_{2}^{k}$ and let the algorithm output the uniform distribution over the strings $\left(\left\langle w, v_{c(1)}\right\rangle, \ldots,\left\langle w, v_{c(n)}\right\rangle\right)$ where $w$ is uniformly chosen from $\mathbb{F}_{2}^{k}$. Observe that every two adjacent vertices $i, j$ are assigned by $c$ to distinct colors, and that this implies that the inner products $\left\langle w, v_{c(i)}\right\rangle$ and $\left\langle w, v_{c(j)}\right\rangle$ are independent. Therefore, the distribution that our algorithm outputs is a linear $G$-wise independent distribution, and its sample space is of size $2^{k}=N+1=\widetilde{O}\left(n^{1-\frac{3}{\ell+1}}\right)$. 
Remark 3.11. For the special case of $\ell=4$ one can use Claim 3.2 to show that $\ell(G)=4$ if and only if $G$ is 3 -colorable. Thus, the best known polynomial-time algorithm for coloring 3-colorable graphs [12, 24, 25] implies that there exists a polynomial-time algorithm that given an $n$ vertex graph $G$ satisfying $\ell(G)=4$ finds a linear $G$-wise independent distribution with sample space of size $o\left(n^{1 / 5}\right)$.

We now relate the minrank parameter (see Definition 2.4) to the $\ell$ parameter.

Lemma 3.12. For every graph $G, \ell(G) \geq \operatorname{minrk}_{\mathbb{R}}(\bar{G})-1$.

Proof. It can be assumed that $G$ contains no isolated vertices, since such vertices do not affect $\ell(G)$ nor $\operatorname{minrk}_{\mathbb{R}}(\bar{G})$. Consider a uniform $G$-wise independent distribution over $\{0,1\}^{n}$ with sample space of size $\ell$, and let $B$ denote an $\ell$ by $n$ Boolean matrix whose columns represent the vertex distributions. Notice that the inner product over $\mathbb{R}$ of every two columns of $B$ that correspond to adjacent vertices in $G$ equals $\frac{\ell}{4}$ and that the inner product over $\mathbb{R}$ of every column of $B$ with itself equals $\frac{\ell}{2}$. Let $J_{n}$ denote the all-one $n$ by $n$ matrix and define $A=B^{T} \cdot B-\frac{\ell}{4} \cdot J_{n}$. Observe that every entry in the diagonal of $A$ equals $\frac{\ell}{4}$, whereas every entry corresponding to an edge of $G$ (i.e., a non-edge of $\bar{G}$ ) equals 0 . This implies that the matrix $A$ represents $\bar{G}$, hence

$$
\operatorname{minrk}_{\mathbb{R}}(\bar{G}) \leq \operatorname{rank}_{\mathbb{R}}(A) \leq \operatorname{rank}_{\mathbb{R}}\left(B^{T} \cdot B\right)+\operatorname{rank}_{\mathbb{R}}\left(\frac{\ell}{4} \cdot J_{n}\right) \leq \ell+1
$$

We turn to show some hardness results on the $\ell$ parameter.

Theorem 3.13. For every constant $\ell \geq 4$ divisible by 4 , the problem of deciding whether for an input graph $G$ there exists a uniform $G$-wise independent distribution with sample space of size $\ell$ is NP-complete.

Proof. It is clear that the problem in the statement lies in NP. For NP-hardness, we employ a result of Hell and Nešetrril [21] on the hardness of the $H$-coloring problem, in which given an input graph $G$ the goal is to decide whether there is a homomorphism from $G$ to the fixed graph $H$. It was shown in [21] that this problem is NP-hard whenever $H$ is not bipartite and does not contain a loop. The graph $G_{\ell}$ (see Definition 3.1) clearly does not contain a loop and we observe that it is not bipartite. To see this, let $u_{1}, u_{2}, u_{3}$ be the three vectors consisting of $\frac{\ell}{4}$ concatenated copies of $(0,0,1,1),(0,1,0,1)$ and $(0,1,1,0)$ respectively, and notice that these vectors form a triangle in $G_{\ell}$. Therefore, the result of [21] implies that deciding whether for an input graph $G$ there is a homomorphism from $G$ to $G_{\ell}$, i.e., there exists a uniform $G$-wise independent distribution with sample space of size $\ell$, is NP-hard.

Finally, we observe that hardness of approximating the $\ell$ parameter can be derived from known hardness results on the chromatic number of a graph. It was shown in $[14,15]$ that assuming a certain variant of the Unique Games Conjecture of [26], it is NP-hard to decide whether the chromatic number of an $n$ vertex input graph is at most some constant or at least $\Omega\left(\log ^{c} n\right)$ for some constant $c>0$. Combining this with Theorem 3.6 implies the following corollary.

Corollary 3.14. Assuming the variant of the Unique Games Conjecture from [14, 15], there exists a constant $c>0$ for which approximating $\ell(G)$ for an $n$ vertex input graph $G$ to within a factor of $c \cdot \log \log n$ is NP-hard. 


\section{$H$-WISE INDEPENDENCE}

\section{Testing $H$-wise Independence}

In this section we present our results on the problem of testing $H$-wise independence. Recall that the goal is to develop an algorithm which given samples from a distribution distinguishes $H$-wise independent distributions from those that are $\delta$-far from any $H$-wise independent distribution (see Definition 1.6). We turn to prove upper and lower bounds on the sample complexity of such an algorithm for a general $k$-uniform hypergraph $H$, extending results of $[5,2]$. We note that for ease of presentation, $k$ is assumed to be a fixed constant and thus we do not explicitly state the (usually exponential) dependence of our expressions on $k$.

\subsection{Upper Bound}

A natural way to test $H$-wise independence is to pick samples from the input distribution and to calculate the biases of the samples on every $S \in \tilde{E}$, where $\tilde{E}$ is the set of all non-empty subsets of edges in $H$. This is a generalization of the tester presented in [2] for testing $k$-wise independence. If the distribution is $H$-wise independent the biases are expected to be close to 0 . For the analysis, one has to show that if the distribution is far from $H$-wise independence then at least one of these biases will be far away from 0 with high probability, and thus one may be able to identify this scenario via multiple queries.

The question of estimating the statistical distance of a general distribution from the class of $k$-wise independent distributions was first addressed in [5]. It was shown there that every distribution $X$ over $\{0,1\}^{n}$ with $\left|\operatorname{bias}_{S}(X)\right|=\varepsilon$ for some $S \subseteq[n]$ can be modified to another distribution $Y$ over $\{0,1\}^{n}$ such that $\operatorname{bias}_{S}(Y)=0$, the other biases do not increase (in absolute value), and the statistical distance between $X$ and $Y$ is at most $\varepsilon$. Applying this argument iteratively to every $S$ such that $1 \leq|S| \leq k$ implies that for every distribution $X$ there exists a $k$-wise independent distribution $Y$ such that $\Delta(X, Y) \leq$ $n^{k} \cdot \max _{1 \leq|S| \leq k}\left|\operatorname{bias}_{S}(X)\right|$. A similar approach can be useful for estimating the distance of a general distribution from the class of $H$-wise independent distributions for some $k$-uniform hypergraph $H$. Here, the modification of [5] should be applied only to every $S \in \tilde{E}$, since if $\operatorname{bias}_{S}(Y)=0$ for every such $S$ then $Y$ is $H$-wise independent (see Lemma 2.1). Hence, for every distribution $X$ there exists some $H$-wise independent distribution $Y$ such that $\Delta(X, Y) \leq|\tilde{E}| \cdot \max _{S \in \tilde{E}}\left|\operatorname{bias}_{S}(X)\right|$. As will be clear from our analysis (and that of [2]), this result implies a tester for $H$-wise independence with query complexity $O\left(|\tilde{E}|^{2}\right)$. We improve on this below.

The bound of [5] on the statistical distance of a distribution from $k$-wise independence was improved in [2]. The high level idea was to approximate a general distribution $X$ by a function $Y$ all of whose Fourier coefficients $\widehat{Y}(S)$ for non-empty $S$ of size at most $k$ are zeros. This was done by defining $Y$ to satisfy $\widehat{Y}(S)=0$ whenever $1 \leq|S| \leq k$ and $\widehat{Y}(S)=\widehat{X}(S)$ otherwise. If $Y$ would be a distribution then it must be $k$ wise independent, and one can prove using Parseval's equality that $\Delta(X, Y) \leq n^{k / 2} \cdot \max _{1 \leq|S| \leq k}\left|\operatorname{bias}_{S}(X)\right|$. This significantly improves upon the bound of [5]. However, it is definitely possible that the function $Y$ is not a distribution, since its values might be negative. In order to overcome this difficulty the approach taken in [5] was to replace the function $Y$ by a convex combination of $Y$ and some $k$-wise independent distributions. Two types of $k$-wise independent distributions were used for this goal: the uniform distribution $U_{\{0,1\}^{n}}$ (whose support size is $2^{n}$ ) which handled the negative values of points with relatively small magnitude; and the economical $k$-wise independent distributions of [3] (whose support size is roughly $n^{\left\lfloor\frac{k}{2}\right\rfloor}$ ) which handled the negative values of points with relatively large magnitude. 
Here we employ the techniques of [2] and generalize their bound. For a $k$-uniform hypergraph $H$, given a distribution $X$ our goal is to find some $H$-wise independent distribution close to $X$. To do so, we consider the function $Y$ whose Fourier coefficients are identical to the ones of $X$, besides the coefficients corresponding to some $S \in \tilde{E}$ for which we require $\widehat{Y}(S)=0$. Then, in order to make $Y$ a distribution, we take a convex combination of $Y$ with certain $H$-wise independent distributions. To handle the points of small magnitude we take, as in [2], the uniform distribution $U_{\{0,1\}^{n}}$ over $n$ bit strings, whereas to handle the points of large magnitude we take uniform $H$-wise distributions of sample space of size $\ell(H)$. Finally, we apply the upper bound on $\ell(H)$ of Schulman [36] (Theorem 1.3) and obtain an upper bound on the distance of $X$ from $H$-wise independence in terms of the number of edges in $H$ and $\max _{S \in \tilde{E}}\left|\operatorname{bias}_{S}(X)\right|$ generalizing that of [2].

Lemma 4.1. For every $n$ vertex $k$-uniform hypergraph $H=([n], E)$ and a distribution $D$ over $\{0,1\}^{n}$,

$$
\Delta\left(D, \mathfrak{D}_{H-\text { wise }}\right) \leq O\left(\log ^{k / 2}|\tilde{E}|\right) \cdot \sqrt{\sum_{S \in \tilde{E}}\left|\operatorname{bias}_{S}(D)\right|^{2}} \leq O\left(|\tilde{E}|^{1 / 2} \cdot \log ^{k / 2}|\tilde{E}|\right) \cdot \max _{S \in \tilde{E}}\left|\operatorname{bias}_{S}(D)\right| .
$$

Proof. Let $D$ be a distribution over $\{0,1\}^{n}$, and write $D=D^{(1)}+D^{(2)}$ where $D^{(1)}, D^{(2)}:\{0,1\}^{n} \rightarrow \mathbb{R}$ are the functions whose Fourier coefficients satisfy

$$
\widehat{D^{(1)}}(S)=\left\{\begin{array}{ll}
0 & \text { if } S \in \tilde{E} \\
\widehat{D}(S) & \text { o.w. }
\end{array}, \quad \widehat{D^{(2)}}(S)= \begin{cases}\widehat{D}(S) & \text { if } S \in \tilde{E} \\
0 & \text { o.w. }\end{cases}\right.
$$

Denote $b_{1}=\sum_{S \in \tilde{E}}\left|\operatorname{bias}_{S}(D)\right|$ and $b_{2}=\sqrt{\sum_{S \in \tilde{E}}\left|\operatorname{bias}_{S}(D)\right|^{2}}$, and observe that $b_{2} \leq b_{1} \leq \sqrt{|\tilde{E}|} \cdot b_{2}$ (the right hand side follows from Cauchy-Schwarz inequality). For some $\lambda$ to be determined later, we consider a partition of $\{0,1\}^{n}$ into two sets: the set $Z=\left\{z \in\{0,1\}^{n} \mid D^{(2)}(z) \geq \frac{\lambda \cdot b_{2}}{2^{n}}\right\}$ of heavy points and the set $\{0,1\}^{n} \backslash Z$ of light points. Let $U_{z}$ be a uniform $H$-wise independent distribution over $\{0,1\}^{n}$ whose sample space contains $z$ and has size $\ell(H)$. Such a distribution can be obtained by taking some optimal uniform $H$-wise independent distribution and xoring its samples with a fixed vector in order to include $z$ in the support. Define a function $D^{\prime}:\{0,1\}^{n} \rightarrow \mathbb{R}$ by

$$
D^{\prime}(x)=\frac{D^{(1)}(x)+\frac{\lambda \cdot b_{2}}{2^{n}}+w \cdot \sum_{z \in Z} U_{z}(x)}{1+\lambda b_{2}+w \cdot|Z|},
$$

where $w=\frac{\ell(H) \cdot b_{1}}{2^{n}}$. We claim that the function $D^{\prime}$ represents a distribution, i.e., its values on $\{0,1\}^{n}$ are non-negative and sum to 1 . Since $\widehat{D^{(1)}}(\phi)=2^{-n}$, it can be seen that $\sum_{x} D^{\prime}(x)=1$, so we turn to show that $D^{\prime}$ is non-negative on light and heavy points separately:

1. Assume that $x$ is light, i.e., $D^{(2)}(x)<\frac{\lambda \cdot b_{2}}{2^{n}}$. By the definition of $D^{\prime}$,

$$
D^{\prime}(x) \geq \frac{D^{(1)}(x)+\frac{\lambda \cdot b_{2}}{2^{n}}}{1+\lambda b_{2}+w \cdot|Z|}=\frac{D(x)-D^{(2)}(x)+\frac{\lambda \cdot b_{2}}{2^{n}}}{1+\lambda b_{2}+w \cdot|Z|} \geq 0 .
$$

2. Assume that $x$ is heavy, i.e., $D^{(2)}(x) \geq \frac{\lambda \cdot b_{2}}{2^{n}}$, and observe that

$$
D^{\prime}(x) \geq \frac{D^{(1)}(x)+w \cdot U_{x}(x)}{1+\lambda b_{2}+w \cdot|Z|}=\frac{D^{(1)}(x)+\frac{w}{\ell(H)}}{1+\lambda b_{2}+w \cdot|Z|}=\frac{D(x)-D^{(2)}(x)+\frac{b_{1}}{2^{n}}}{1+\lambda b_{2}+w \cdot|Z|} \geq 0
$$


where the last inequality holds since any $x \in\{0,1\}^{n}$ satisfies

$$
\left|D^{(2)}(x)\right|=\left|\sum_{S \in \tilde{E}} \widehat{D}(S) \chi_{S}(x)\right| \leq \sum_{S \in \tilde{E}}|\widehat{D}(S)|=2^{-n} \cdot b_{1} .
$$

Moreover, by Lemma 2.1, the distribution $D^{\prime}$ is $H$-wise independent as $\operatorname{bias}_{S}\left(D^{\prime}\right)=2^{n} \cdot \widehat{D}^{\prime}(S)$ is a linear combination of $\widehat{D^{(1)}}(S)$ and the $\widehat{U}_{z}(S)$ which are all zeros for any $S \in \tilde{E}$.

Now, apply Cauchy-Schwarz inequality and Parseval's equality to get that

$$
\left\|D-D^{(1)}\right\|_{1}=2^{-n} \cdot \sum_{x \in\{0,1\}^{n}}\left|D(x)-D^{(1)}(x)\right| \leq 2^{-n / 2} \cdot \sqrt{\sum_{x \in\{0,1\}^{n}}\left|D(x)-D^{(1)}(x)\right|^{2}}=2^{-n} \cdot b_{2} .
$$

In addition, by the triangle inequality and the fact that the statistical distance between two distributions is always at most 1 , we get that

$$
\left\|D^{(1)}-D^{\prime}\right\|_{1} \leq \lambda \cdot b_{2}\left\|D^{\prime}-U_{\{0,1\}^{n}}\right\|_{1}+w \sum_{z \in Z}\left\|D^{\prime}-U_{z}\right\|_{1} \leq 2^{-n+1} \cdot\left(\lambda \cdot b_{2}+w \cdot|Z|\right) .
$$

This implies that

$$
\Delta\left(D, \mathfrak{D}_{H-\text { wise }}\right) \leq \Delta\left(D, D^{\prime}\right) \leq 2^{n-1} \cdot\left(\left\|D-D^{(1)}\right\|_{1}+\left\|D^{(1)}-D^{\prime}\right\|_{1}\right) \leq \frac{b_{2}}{2}+\lambda \cdot b_{2}+w \cdot|Z| .
$$

It remains to present an upper bound on the number $|Z|$ of heavy points. For this purpose we use the Beckner-Bonami hyper-contractive inequality (Theorem 2.5; [7, 10]). Since Parseval's equality implies that $\left\|\frac{2^{n}}{b_{2}} \cdot D^{(2)}\right\|_{2}=1$, we get that for every even $p>2$,

$$
\frac{|Z|}{2^{n}}=\operatorname{Pr}_{x \in\{0,1\}^{n}}\left[D^{(2)}(x) \geq \frac{\lambda \cdot b_{2}}{2^{n}}\right] \leq \operatorname{Pr}_{x \in\{0,1\}^{n}}\left[\left|\frac{2^{n}}{b_{2}} \cdot D^{(2)}(x)\right| \geq \lambda\right] \leq \frac{\left\|\frac{2^{n}}{b_{2}} \cdot D^{(2)}\right\|_{p}^{p}}{\lambda^{p}} \leq \frac{(p-1)^{p k / 2}}{\lambda^{p}} .
$$

Finally, we choose the parameters $p=\log (\ell(H) \cdot \sqrt{|\tilde{E}|})$ (which can be assumed to be even) and $\lambda=2 p^{k / 2}$ and obtain that

$$
|Z| \leq 2^{n} \cdot\left(\frac{p^{k / 2}}{\lambda}\right)^{p} \leq \frac{2^{n}}{\ell(H) \cdot \sqrt{|\tilde{E}|}}
$$

Combining this bound with (4.1) we get

$$
\Delta\left(D, \mathfrak{D}_{H-\text { wise }}\right) \leq \frac{b_{2}}{2}+\lambda \cdot b_{2}+\frac{\ell(H) \cdot b_{1}}{2^{n}} \cdot|Z| \leq b_{2}\left(\frac{3}{2}+\lambda\right)
$$

By Theorem 1.3, $\ell(H) \leq 2^{k+1} \cdot|E|$, so it follows that $\Delta\left(D, \mathfrak{D}_{H-\text { wise }}\right) \leq O\left(\log ^{k / 2}|\tilde{E}|\right) \cdot b_{2}$.

Equipped with Lemma 4.1, we are ready to prove our upper bound on the sample complexity of testing $H$-wise independence.

Theorem 4.2. For every $\delta>0$ and an $n$ vertex $k$-uniform hypergraph $H=([n], E)$ there exists $a \delta$-tester for $H$-wise independence with sample complexity $O\left(\frac{|E| \cdot \log ^{k+1}|E|}{\delta^{2}}\right)$. 
Proof. Following [2], consider the tester which draws a set $Q$ of $q=O\left(\frac{|\tilde{E}| \cdot \log ^{k+1}|\tilde{E}|}{\delta^{2}}\right)$ independent samples from the input distribution $D$ and accepts if and only if $\left|\operatorname{bias}_{S}(Q)\right|<\delta^{\prime}$ for every $S \in \tilde{E}$ where $\delta^{\prime}=\frac{c \cdot \delta}{|\tilde{E}|^{1 / 2} \cdot \log ^{k / 2}|\tilde{E}|}$ for a certain absolute constant $c$.

Assume that $D$ is $H$-wise independent. By the Chernoff bound, the probability that $\left|\operatorname{bias}_{S}(Q)\right| \geq 2 \delta^{\prime}$ is at most $e^{-\Omega\left(q \delta^{2}\right)} \leq \frac{1}{3|\tilde{E}|}$, so by the union bound the probability that the tester rejects is at most $\frac{1}{3}$. On the other hand, assume that $\Delta\left(D, \mathfrak{D}_{H-\text { wise }}\right) \geq \delta$, so by Lemma 4.1 (for a certain choice of $c$ ) there exists some $S \in \tilde{E}$ such that $\left|\operatorname{bias}_{S}(D)\right| \geq 2 \delta^{\prime}$. For this $S$, by the Chernoff bound,

$$
\operatorname{Pr}\left[\left|\operatorname{bias}_{S}(Q)\right|<\delta^{\prime}\right] \leq \operatorname{Pr}\left[\left|\operatorname{bias}_{S}(Q)-\operatorname{bias}_{S}(D)\right| \geq \delta^{\prime}\right] \leq e^{-\Omega\left(q \delta^{\prime 2}\right)} \leq \frac{1}{3},
$$

and with such probability the tester accepts. Since $|\tilde{E}| \leq 2^{k} \cdot|E|$ the result follows.

\subsection{Lower Bounds}

A standard method to prove lower bounds on the sample complexity of testing algorithms is to present two input distributions, such that one of them should be accepted and the other one should be rejected, however it is impossible to distinguish one from another with few samples. We turn to show a lower bound for testing $H$-wise independence using this method. To this end, we present a lower bound on the min-entropy of any $H$-wise independent distribution. Our bound, depending on the number $|\bar{E}|$ of non-edges of $H$, is given in the following lemma and generalizes a statement of [5] on $k$-wise independence.

Lemma 4.3. For every even $k$ and every $n$ vertex $k$-uniform hypergraph $H=([n], E)$, every $H$-wise independent distribution over $\{0,1\}^{n}$ has min-entropy at least $\log _{2}\left(\frac{n^{k}}{2^{2 k} \cdot|\bar{E}|+(k / 2)^{k} \cdot n^{k / 2}}\right)$. The same holds for an odd $k$ replacing $k$ by $k-1$ in the bound.

Proof. We prove the lemma for an even $k$, and it can be similarly proven for an odd $k$. Let $D=\left(D_{1}, \ldots, D_{n}\right)$ be some $H$-wise independent distribution and assume without loss of generality that the all-zero string has the maximum probability according to $D$ (otherwise xor $D$ with the string that has maximum probability). Define $Z_{i}=D_{i}-\frac{1}{2}$ and apply the generalized Chebyshev inequality to obtain

$$
\operatorname{Pr}[D=(0, \ldots, 0)]=\operatorname{Pr}\left[\sum_{i=1}^{n} D_{i}=0\right] \leq \operatorname{Pr}\left[\left|\sum_{i=1}^{n} Z_{i}\right| \geq \frac{n}{2}\right] \leq \frac{\mathbf{E}\left[\left(\sum_{i=1}^{n} Z_{i}\right)^{k}\right]}{(n / 2)^{k}}
$$

Write $\mathbf{E}\left[\left(\sum_{i=1}^{n} Z_{i}\right)^{k}\right]=\sum_{i_{1}, \ldots, i_{k} \in[n]} \mathbf{E}\left[Z_{i_{1}} \cdots Z_{i_{k}}\right]$ and consider a term $Z_{i_{1}} \cdots Z_{i_{k}}$ for which $\left\{i_{1}, \ldots, i_{k}\right\}$ is an edge in $H$ or a subset of an edge in $H$. Observe that if there is at least one index $i_{j}$ that appears exactly once in this term then the expectation of this term contributes 0 to the sum. Indeed, in this case $\mathbf{E}\left[Z_{i_{1}} \cdots Z_{i_{k}}\right]=\mathbf{E}\left[Z_{i_{j}}\right] \cdot \mathbf{E}\left[\Pi_{h \neq j} Z_{i_{h}}\right]=0$. Therefore, the only terms which might have positive contribution to the sum are the ones that correspond to a subset of a non-edge, whose number is smaller than $\left(\begin{array}{c}2 k-1 \\ k\end{array}\right) \cdot|\bar{E}|<2^{2 k} \cdot|\bar{E}|$, and the ones in which each index appears at least twice, whose number is smaller than $\left(\begin{array}{c}n \\ k / 2\end{array}\right) \cdot(k / 2)^{k}<(k / 2)^{k} \cdot n^{k / 2}$. Observe that every term in the sum contributes at most $\frac{1}{2^{k}}$ since for every $i,\left|Z_{i}\right| \leq \frac{1}{2}$. Hence, (4.2) implies that the maximum probability of a string according to the 
distribution $D$ is at most

$$
\frac{\frac{1}{2^{k}} \cdot\left(2^{2 k} \cdot|\bar{E}|+(k / 2)^{k} \cdot n^{k / 2}\right)}{(n / 2)^{k}}=\frac{2^{2 k} \cdot|\bar{E}|+(k / 2)^{k} \cdot n^{k / 2}}{n^{k}},
$$

and we are done.

Equipped with Lemma 4.3 we are ready to prove the following lower bound.

Theorem 4.4. For every even $k$, $n$ vertex $k$-uniform hypergraph $H$ and $\delta \leq \frac{1}{2}$, $\delta$-testing $H$-wise independence requires at least $\Omega\left(\sqrt{\frac{n^{k}}{|\bar{E}|+n^{k / 2}}}\right)$ samples from the distribution. The same holds for an odd $k$ replacing $k$ by $k-1$ in the bound.

Proof. We prove the theorem for an even $k$, and it can be similarly proven for an odd $k$. Denote $z=\frac{n^{k}}{2^{2 k} \cdot|\bar{E}|+(k / 2)^{k} \cdot n^{k / 2}}$, let $A$ be a tester which makes at most $q=o(\sqrt{z})$ queries, and consider the following two distributions: the uniform distribution $U_{\{0,1\}^{n}}$ and a uniform distribution $D_{Q}$ over some uniformly chosen set $Q \subseteq\{0,1\}^{n}$ of $\frac{z}{2}$ strings. Whereas the former is clearly $H$-wise independent distribution, it turns out that the latter is $\frac{1}{2}$-far from any such distribution. Indeed, by Lemma 4.3 any $H$-wise independent distribution $D$ has min-entropy at least $\log _{2} z$, hence

$$
\Delta\left(D, D_{Q}\right) \geq \operatorname{Pr}\left[D \in\{0,1\}^{n} \backslash \operatorname{support}\left(D_{Q}\right)\right] \geq 1-\frac{\left|\operatorname{support}\left(D_{Q}\right)\right|}{z}=\frac{1}{2} .
$$

By the birthday paradox, all the $q$ strings queried by $A$ from either $U_{\{0,1\}^{n}}$ or $D_{Q}$ are distinct with probability $1-o(1)$, and hence $A$ cannot distinguish between the two with probability bounded away from $\frac{1}{2}$. This contradicts the fact that $A \delta$-tests $H$-wise independence.

Our next lower bound holds for $\delta=O\left(\frac{1}{n}\right)$ and employs a proof technique used in [2] for a lower bound on the sample complexity of testing $k$-wise independence. At the heart of its proof lies the following lemma which gives a lower bound on the distance of a random uniform distribution from $H$-wise independence.

Lemma 4.5. There exists a constant $c>0$ such that for every $n$ vertex $k$-uniform hypergraph $H=([n], E)$ and $\delta>0$ such that $\delta \leq \frac{1}{2 n}$ the following holds. If $D_{Q}$ is a uniform distribution over some uniformly chosen multiset $Q \subseteq\{0,1\}^{n}$ of size $z=\frac{2^{k} \cdot|E|}{\delta^{2} \cdot n}$ then almost surely $\Delta\left(D_{Q}, \mathfrak{D}_{H-\text { wise }}\right) \geq c \cdot \delta$.

Theorem 4.6. For every $n$ vertex $k$-uniform hypergraph $H=([n], E)$ and $\delta=O\left(\frac{1}{n}\right)$, $\delta$-testing $H$-wise independence requires at least $\Omega\left(\frac{1}{\delta} \cdot \sqrt{\frac{|E|}{n}}\right)$ samples from the distribution.

Proof. Define $z=\frac{c^{2} \cdot 2^{k} \cdot|E|}{\delta^{2} \cdot n}$ for some $\delta \leq \frac{c}{2 n}$, where $c$ is the constant from Lemma 4.5. Let $A$ be a tester which makes at most $q=o(\sqrt{z})$ queries, and consider the following two distributions: the uniform distribution $U_{\{0,1\}^{n}}$ and a uniform distribution $D_{Q}$ over some uniformly chosen multiset $Q \subseteq\{0,1\}^{n}$ of $z$ strings. Whereas the former is clearly $H$-wise independent distribution, by Lemma 4.5 the latter is almost surely $\delta$-far from any such distribution. By the birthday paradox, all the strings queried by $A$ from either $U_{\{0,1\}^{n}}$ or $D_{Q}$ are distinct with probability $1-o(1)$, and hence $A$ cannot distinguish between the two with probability bounded away from $\frac{1}{2}$. This contradicts the fact that $A \delta$-tests $H$-wise independence. 
We need the following concentration result.

Theorem 4.7 (Bernstein's Inequality [9]). Let $X_{1}, \ldots, X_{z}$ be independent real-valued random variables such that $\left|X_{i}\right| \leq C$ for all $1 \leq i \leq z$. Let $\mu=\sum_{i=1}^{z} \mathbf{E}\left[X_{i}\right]$ and $\sigma^{2}=\frac{1}{z} \cdot \sum_{i=1}^{z} \operatorname{Var}\left[X_{i}\right]$. Then, for any $t>0$,

$$
\operatorname{Pr}\left[\left|\sum_{i=1}^{z} X_{i}-\mu\right|>z t\right] \leq e^{-\frac{z t^{2}}{2 \sigma^{2}+2 C t / 3}}
$$

of Lemma 4.5. Let $D$ be some $H$-wise independent distribution over $\{0,1\}^{n}$ with minimum statistical distance from $D_{Q}$, and define $f_{Q}=D_{Q}-D$. Notice that by Lemma 2.1 for every $S \in \tilde{E}, \widehat{f_{Q}}(S)=\widehat{D_{Q}}(S)$ and that $\Delta\left(D_{Q}, \mathfrak{D}_{H-\text { wise }}\right)=2^{n-1} \cdot\left\|f_{Q}\right\|_{1}$. Let $g_{Q}, h_{Q}:\{0,1\}^{n} \rightarrow \mathbb{R}$ be the functions defined by

$$
\widehat{g_{Q}}(S)=\left\{\begin{array}{ll}
\widehat{f_{Q}}(S) & \text { if } S \in \tilde{E} \\
0 & \text { o.w. }
\end{array}, \quad h_{Q}=f_{Q} * g_{Q} .\right.
$$

Observe that $\widehat{h_{Q}}(S)=\widehat{f_{Q}}(S)^{2}$ if $S \in \tilde{E}$ and $\widehat{h_{Q}}(S)=0$ otherwise.

We now prove that

1. for some $c^{\prime}>0,\left\|h_{Q}\right\|_{\infty} \geq c^{\prime} \cdot \frac{|\tilde{E}|}{2^{2 n} \cdot z}$ almost surely, and

2. for some $c^{\prime \prime}>0,\left\|g_{Q}\right\|_{\infty} \leq \frac{c^{\prime \prime}}{2^{n}} \cdot \sqrt{\frac{n \cdot|\tilde{E}|}{z}}$ almost surely.

By the union bound both (1) and (2) hold almost surely. Therefore, by Young's inequality, we obtain that for some $c>0$ almost surely

$$
\Delta\left(D_{Q}, \mathfrak{D}_{H-\text { wise }}\right)=2^{n-1} \cdot\left\|f_{Q}\right\|_{1} \geq 2^{n-1} \cdot \frac{\left\|h_{Q}\right\|_{\infty}}{\left\|g_{Q}\right\|_{\infty}} \geq c \cdot \delta
$$

where for the last inequality we used $|\tilde{E}| \leq 2^{k} \cdot|E|$.

Proof of Item (1). First, observe that $\left\|h_{Q}\right\|_{\infty}=h_{Q}(0)$, since for every $x \in\{0,1\}^{n}$,

$$
\left|h_{Q}(x)\right|=\left|\sum_{S \in \tilde{E}} \widehat{h_{Q}}(S) \chi_{S}(x)\right| \leq \sum_{S \in \tilde{E}}\left|\widehat{h_{Q}}(S)\right|=\sum_{S \in \tilde{E}} \widehat{h_{Q}}(S)=h_{Q}(0) .
$$

This implies that $\left\|h_{Q}\right\|_{\infty}=h_{Q}(0)=\sum_{S \in \tilde{E}} \widehat{f_{Q}}(S)^{2}$, where for every $S \in \tilde{E}$,

$$
\widehat{f_{Q}}(S)=\widehat{D_{Q}}(S)=\frac{1}{2^{n}} \cdot \sum_{y \in Q} D_{Q}(y) \cdot \chi_{S}(y)=\frac{1}{2^{n} z} \cdot \sum_{y \in Q} \chi_{S}(y) .
$$

A calculation of the expectation and the variance of $\left\|h_{Q}\right\|_{\infty}$ (similar to the calculation in [2]) implies that

$$
\mathbf{E}_{Q}\left[\left\|h_{Q}\right\|_{\infty}\right]=\frac{|\tilde{E}|}{2^{2 n} z} \cdot\left(1-\frac{z-1}{2^{n}-1}\right) \quad \text { and } \quad \operatorname{Var}_{Q}\left[\left\|h_{Q}\right\|_{\infty}\right]=\frac{2|\tilde{E}|}{2^{4 n} z^{2}}(1-o(1)) .
$$

By Chebyshev's inequality, it follows that almost surely $\left\|h_{Q}\right\|_{\infty} \geq c^{\prime} \cdot \mathbf{E}_{Q}\left[\left\|h_{Q}\right\|_{\infty}\right]$ for some constant $c^{\prime}>0$, and we are done. 
Proof of Item (2). Fix $x \in\{0,1\}^{n}$, and define the function $Y_{x}$ by $Y_{x}(y)=\sum_{S \in \tilde{E}} \chi_{S}(x+y)$. Using (4.3) we observe that

$$
g_{Q}(x)=\sum_{S \in \tilde{E}} \widehat{f_{Q}}(S) \chi_{S}(x)=\frac{1}{2^{n} z} \cdot \sum_{S \in \tilde{E}} \sum_{y \in Q} \chi_{S}(x+y)=\frac{1}{2^{n} z} \cdot \sum_{y \in Q} Y_{x}(y) .
$$

Notice that the random variables $\left\{Y_{x}(y)\right\}_{y \in Q}$ are independent and satisfy $\left|Y_{x}(y)\right| \leq|\tilde{E}|$. In addition, since the expectation of $\chi_{S}(y)$ over a random uniformly chosen $y$ for a non-empty $S$ is 0 , it can be observed that $\mathbf{E}_{y}\left[Y_{x}(y)\right]=0$ and that $\operatorname{Var}_{y}\left[Y_{x}(y)\right]=|\tilde{E}|$. Define $t=2 \cdot \sqrt{\frac{n|\tilde{E}|}{z}}$ and notice that $t \leq 1$ by the definition of $z$ and the assumption on $\delta$. Apply Bernstein's inequality (Theorem 4.7) to get that

$$
\operatorname{Pr}\left[\left|g_{Q}(x)\right|>\frac{t}{2^{n}}\right]=\operatorname{Pr}\left[\left|\sum_{y \in Q} Y_{x}(y)\right|>z t\right] \leq e^{-\frac{z t^{2}}{2|\tilde{E}|+2|\tilde{E}| t / 3}} \leq e^{-\frac{z t^{2}}{3|\tilde{E}|}}=o\left(2^{-n}\right) .
$$

By the union bound, $\left|g_{Q}(x)\right| \leq \frac{t}{2^{n}}$ for every $x \in\{0,1\}^{n}$ almost surely, hence for some constant $c^{\prime \prime}>0$, almost surely $\left\|g_{Q}\right\|_{\infty} \leq \frac{c^{\prime \prime}}{2^{n}} \cdot \sqrt{\frac{n|\tilde{E}|}{z}}$.

\section{Acknowledgments}

We would like to thank Leonard Schulman and Adi Shraibman for helpful discussions, Ning Xie for clarifications on [2], and the anonymous reviewers for their valuable suggestions.

\section{References}

[1] Noga Alon: A parallel algorithmic version of the local lemma. Random Struct. Algorithms, 2(4):367-378, 1991. Preliminary version in FOCS'91. 2

[2] Noga Alon, Alexandr Andoni, Tali Kaufman, Kevin Matulef, Ronitt Rubinfeld, AND NING XIE: Testing $k$-wise and almost $k$-wise independence. In STOC, pp. 496-505, 2007. 5 , $6,13,14,16,17,18,19$

[3] Noga Alon, LÁszló Babai, And Alon Itai: A fast and simple randomized parallel algorithm for the maximal independent set problem. J. Algorithms, 7(4):567-583, 1986. 2, 3, 4, 13

[4] Noga Alon, Oded Goldreich, Johan Håstad, and René Peralta: Simple construction of almost $k$-wise independent random variables. Random Struct. Algorithms, 3(3):289-304, 1992. Preliminary version in FOCS' 90.2

[5] Noga Alon, Oded Goldreich, and Yishay Mansour: Almost $k$-wise independence versus $k$-wise independence. Inf. Process. Lett., 88(3):107-110, 2003. 5, 6, 13, 16

[6] Ziv BAR-Yossef, Yitzhak Birk, T. S. JAYRAM, AND TOMER Kol: Index coding with side information. In FOCS, pp. 197-206, 2006. 8 
[7] W. BeCKner: Inequalities in Fourier analysis. Ann. of Math., 102(1):159-182, 1975. 9, 15

[8] Bonnie Berger And John Rompel: Simulating $\left(\log ^{c} n\right)$-wise independence in NC. J. ACM, 38(4):1026-1046, 1991. Preliminary version in FOCS'89. 2

[9] Sergei Bernstein: The Theory of Probabilities. Gostehizdat Publishing House, Moscow, 1946. 18

[10] A. Bonami: Étude des coefficients de Fourier des fonctions de $L^{p}(G)$. Annales de l'Institut Fourier, 20(2):335-402, 1970. 9, 15

[11] Joan Boyar, Gilles Brassard, and René Peralta: Subquadratic zero-knowledge. J. ACM, 42(6):1169-1193, 1995. Preliminary version in FOCS'91. 2

[12] Eden Chlamtac: Approximation algorithms using hierarchies of semidefinite programming relaxations. In FOCS, pp. 691-701, 2007. 12

[13] Benny Chor, Oded Goldreich, Johan Håstad, Joel Friedman, Steven Rudich, and ROMAN SMOLENSKY: The bit extraction problem of $t$-resilient functions. In FOCS, pp. 396-407, 1985. 2, 3, 4

[14] Irit Dinur, Elchanan Mossel, And Oded Regev: Conditional hardness for approximate coloring. SIAM J. Comput., 39(3):843-873, 2009. Preliminary version in STOC'06. 4, 12

[15] IRIT DinUR AND IGOR SHINKAR: On the conditional hardness of coloring a 4-colorable graph with super-constant number of colors. In APPROX-RANDOM, pp. 138-151, 2010. 4, 12

[16] PAUl ERdös: Problems and results in graph theory and combinatorial analysis. In Proceedings of the Fifth British Combinatorial Conference, pp. 169-192, 1975. 4, 10

[17] URIEL FeIGE: Randomized graph products, chromatic numbers, and the Lovász $\vartheta$-function. Combinatorica, 17(1):79-90, 1997. Preliminary version in STOC'95. 11

[18] Peter Frankl and Vojtěch Rödl: Forbidden intersections. Trans. Amer. Math. Soc., 300(1):259-286, 1987. 4, 10

[19] Willem HaEMERS: On some problems of Lovász concerning the Shannon capacity of a graph. IEEE Trans. Inform. Theory, 25(2):231-232, 1979. 5

[20] Willem Haemers: An upper bound for the Shannon capacity of a graph. In Algebraic methods in graph theory, Vol. I, II (Szeged, 1978), volume 25 of Colloq. Math. Soc. János Bolyai, pp. 267-272. North-Holland, Amsterdam, 1981. 5, 8

[21] Pavol Hell and Jaroslav NeŠEtřIl: On the complexity of $H$-coloring. J. Comb. Theory, Ser. $B, 48(1): 92-110,1990.4,12$

[22] David R. Karger, Rajeev Motwani, And Madhu Sudan: Approximate graph coloring by semidefinite programming. J. ACM, 45(2):246-265, 1998. Preliminary version in FOCS'94. 5, 8, 11 


\section{$H$-WISE INDEPENDENCE}

[23] Richard M. KARP AND Avi Wigderson: A fast parallel algorithm for the maximal independent set problem. J. ACM, 32(4):762-773, 1985. Preliminary version in STOC'84. 2

[24] Ken-ichi Kawarabayashi and Miknel Thorup: Combinatorial coloring of 3-colorable graphs. In FOCS, pp. 68-75, 2012. 12

[25] Ken-IChi Kawarabayashi and Mikkel Thorup: Coloring 3-colorable graphs with less than $n^{1 / 5}$ colors. J. ACM, 64(1):4:1-4:23, 2017. Preliminary version in STACS'14. 12

[26] Subhash Кнот: On the power of unique 2-prover 1-round games. In STOC, pp. 767-775, 2002. 5,12

[27] Donald E. KNuth: The sandwich theorem. Electronic J. Combinatorics, 1:1, 1994. 8

[28] Daphne Koller and Nimrod Megiddo: Constructing small sample spaces satisfying given constraints. SIAM J. Discrete Math., 7(2):260-274, 1994. Preliminary version in STOC'93. 2

[29] LÁszló LovÁsz: On the Shannon capacity of a graph. IEEE Trans. Inform. Theory, 25(1):1-7, 1979. $5,8,11$

[30] Michael Luby: A simple parallel algorithm for the maximal independent set problem. SIAM J. Comput., 15(4):1036-1053, 1986. 2

[31] Michael Luby: Removing randomness in parallel computation without a processor penalty. $J$. Comput. System Sci., 47(2):250-286, 1993. 2

[32] Michael Luby and Avi Wigderson: Pairwise independence and derandomization. Foundations and Trends in Theoretical Computer Science, 1(4), 2005. 2

[33] Rajeev Motwani, Joseph NaOR, And Moni Naor: The probabilistic method yields deterministic parallel algorithms. J. Comput. System Sci., 49(3):478-516, 1994. Preliminary version in FOCS' 89.2

[34] Joseph NaOR AND MONi NAOR: Small-bias probability spaces: Efficient constructions and applications. SIAM J. Comput., 22(4):838-856, 1993. Preliminary version in STOC'90. 2

[35] RonitT Rubinfeld AND Ning XIE: Testing non-uniform $k$-wise independent distributions over product spaces. In ICALP (1), pp. 565-581, 2010. 5

[36] LeOnARD J. Schulman: Sample spaces uniform on neighborhoods. In STOC, pp. 17-25, 1992. $2,3,4,14$

[37] Claude E. Shannon: The zero error capacity of a noisy channel. Institute of Radio Engineers, Transactions on Information Theory, IT-2(September):8-19, 1956. 5

[38] Mario Szegedy: The Lovász local lemma - A survey. In Computer Science - Theory and Applications - 8th International Computer Science Symposium in Russia, CSR, pp. 1-11, 2013. 3

[39] Ning XIE: Personal communication, 2012. 6 


\section{AUTHORS}

Ishay Haviv

School of Computer Science

The Academic College of Tel Aviv-Yaffo

Tel Aviv 61083, Israel

Michael Langberg

Dept. of Electrical Engineering

University at Buffalo

Buffalo, NY, USA 